\title{
A modular cloning toolkit for genome editing in plants
}

\author{
Florian Hahn ${ }^{1}$ (D), Andrey Korolev ${ }^{1,2}$, Laura Sanjurjo Loures ${ }^{1}$ (D) and Vladimir Nekrasov ${ }^{1 *}$ (D)
}

\begin{abstract}
Background: CRISPR/Cas has recently become a widely used genome editing tool in various organisms, including plants. Applying CRISPR/Cas often requires delivering multiple expression units into plant and hence there is a need for a quick and easy cloning procedure. The modular cloning (MoClo), based on the Golden Gate (GG) method, has enabled development of cloning systems with standardised genetic parts, e.g. promoters, coding sequences or terminators, that can be easily interchanged and assembled into expression units, which in their own turn can be further assembled into higher order multigene constructs.

Results: Here we present an expanded cloning toolkit that contains 103 modules encoding a variety of CRISPR/Casbased nucleases and their corresponding guide RNA backbones. Among other components, the toolkit includes a number of promoters that allow expression of CRISPR/Cas nucleases (or any other coding sequences) and their guide RNAs in monocots and dicots. As part of the toolkit, we present a set of modules that enable quick and facile assembly of tRNA-sgRNA polycistronic units without a PCR step involved. We also demonstrate that our tRNAsgRNA system is functional in wheat protoplasts.

Conclusions: We believe the presented CRISPR/Cas toolkit is a great resource that will contribute towards wider adoption of the CRISPR/Cas genome editing technology and modular cloning by researchers across the plant science community.
\end{abstract}

Keywords: CRISPR, Cas9, Plant, Genome editing, Golden Gate, MoClo

\section{Background}

The CRISPR/Cas technology has recently become an easily accessible genome editing tool for many organisms, including plants [1]. Generating gene knockouts has become a rather straightforward CRISPR/Cas application in many plant systems [2-4], while more sophisticated applications, such as allele replacements or targeted gene insertions, still remain a challenge due to low efficiency of homology-directed repair (HDR) in plants [5].

In its conventional form, the CRISPR/Cas system includes a DNA nuclease, such as Cas9, which is guided to a specific genomic location by the guide RNA. Therefore, in

\footnotetext{
* Correspondence: vladimir.nekrasov@rothamsted.ac.uk

${ }^{1}$ Plant Sciences Department, Rothamsted Research, Harpenden AL5 2JQ, UK Full list of author information is available at the end of the article
}

order to perform targeted mutagenesis in planta, one needs to co-express both the CRISPR/Cas nuclease and its cognate guide RNA. Usually, the gene encoding the CRISPR/Cas nuclease is expressed using an RNA polymerase II (Pol II) promoter (e.g. 35Sp), while the guide RNA is expressed under an RNA polymerase III (Pol III) promoter (e.g. U6p or U3p), which has a defined transcription start nucleotide ('G' for U6p or 'A' for U3p). One of the advantages of CRISPR/Cas is multiplexing i.e. one can target DNA at multiple genomic locations by coexpressing multiple guide RNAs specific to those loci. Guide RNAs can be expressed either as individual transcriptional units, each under its own Pol III promoter [4], or as a tRNA-sgRNA polycistronic transcript [6]. In the latter case, guide RNAs are interspaced with tRNAs in a single transcript that gets processed into individual guide

(c) The Author(s). 2020 Open Access This article is licensed under a Creative Commons Attribution 4.0 International License, which permits use, sharing, adaptation, distribution and reproduction in any medium or format, as long as you give appropriate credit to the original author(s) and the source, provide a link to the Creative Commons licence, and indicate if changes were made. The images or other third party material in this article are included in the article's Creative Commons. licence, unless indicated otherwise in a credit line to the material. If material is not included in the article's Creative Commons licence and your intended use is not permitted by statutory regulation or exceeds the permitted use, you will need to obtain permission directly from the copyright holder. To view a copy of this licence, visit http://creativecommons.org/licenses/by/4.0/ The Creative Commons Public Domain Dedication waiver (http://creativecommons.org/publicdomain/zero/1.0/) applies to the data made available in this article, unless otherwise stated in a credit line to the data. 
RNAs by the highly conserved tRNA processing machinery inside the plant cell [6].

As genome editing applications in plants often rely on delivering multiple expression units into plant cells, including a selectable marker, a CRISPR/Cas nucleaseencoding gene and one or more guide RNAs, it is important to be able to assemble DNA constructs encoding such expression units easily and rapidly. The modular cloning (MoClo) system based on the Golden Gate (GG) cloning method [7] is highly flexible and versatile, and provides a means for quick and facile assembly of multiexpression unit constructs using standard genetic parts, such as promoters, terminators, coding sequences etc. The system has already been successfully used for genome editing applications in plants $[3,4,8-10]$ but lacks modules encoding many of the newest genome editing reagents. Here we report on an expanded GG cloning toolkit for genome editing applications in monocot and dicot plants. We believe the toolkit will become a valuable addition to already existing GG-based tools for plant genome editing and be widely used by plant researchers across the community.

\section{Results}

During this study we have generated a set of 103 GG modules that enable one to perform genome editing in both monocot and dicot plant species (Additional file 2: Table S5). The cloning toolkit is an addition to previously published GG modules [7, 9-11] and includes, among many new elements, modules encoding FnCas12a, LbCas12a, Cms1 nucleases, C-G to T-A and A-T to $\mathrm{G}-\mathrm{C}$ base editors, Cas9 variants with alternative protospacer adjacent motif (PAM) specificities (SaCas9, StCas9, ScCas9 etc), Pol II and Pol III promoters, as well as guide RNA backbone modules. The latter enable insertion of the guide sequence by cloning in a pair of annealed complimentary oligos without a PCR amplification step involved. The toolkit enables assembly of CRISPR/Cas constructs that target a single as well as multiple targets with guide RNAs expressed either under individual Pol III promoters (Fig. 1) or using a polycistronic tRNA-sgRNA construct (Fig. 4). With the tRNAsgRNA system proven to be efficient in both monocot (rice, wheat) [6, 12] and dicot (Arabidopsis) [13] plant species, our modules enabling a straightforward PCR-

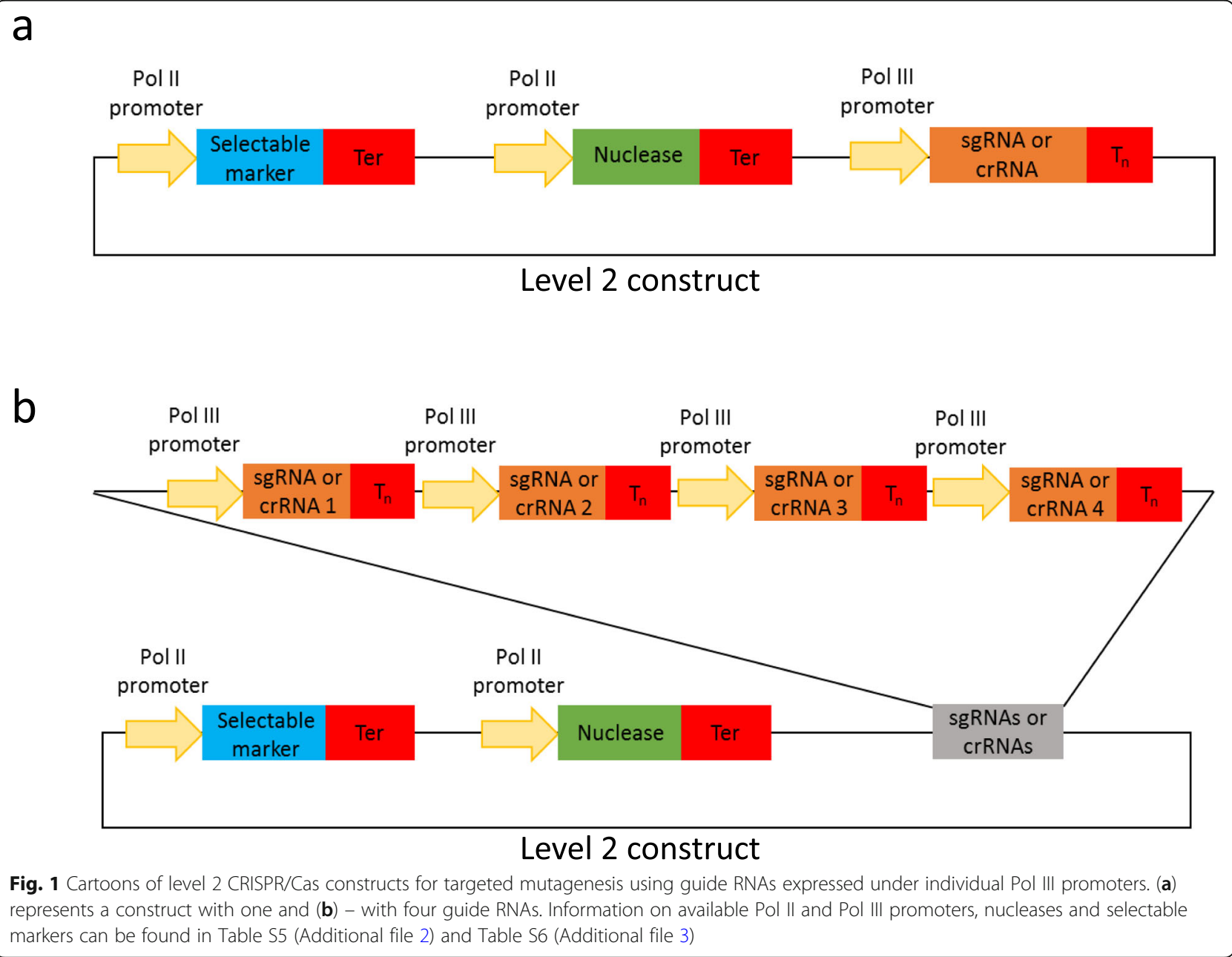


free assembly of polycistronic tRNA-sgRNA arrays for simultaneous editing of multiple genomic targets are one of the highlights of the toolkit.

\section{CRISPR/Cas nuclease modules}

The CRISPR/Cas nuclease level 0 modules include Streptococcus pyogenes Cas9 (SpCas9; pFH13, pFH24 and $\mathrm{pFH} 25$ ) as well as Cas9 variants coming from other bacterial species: Staphylococcus aureus (SaCas9; pFH14), Streptococcus thermophilus (StCas9; pFH15) and Streptococcus canis (ScCas9; pFH76) (Additional file 2: Table S5). SpCas9 [14], with the 'NGG' PAM, is the most commonly used Cas9 variant for genome editing applications in various organisms, including plants. SaCas9 ('NNGRRT' PAM) and StCas9 ('NNRGAA' PAM) are less common but have also been successfully used in rice, tobacco [15] and Arabidopsis [16-18]. ScCas9 (pFH76), SpCas9-NG (pFH32) and SpCas9derived xCas9 (pFH22) are characterised by broadened PAM motif requirements: 'NNG' for ScCas9 [19], and 'NG' for SpCas9-NG [20] and xCas9 [21]. We have also included modules with Cas12a (Cpf1) CRISPR nucleases from Francisella novicida (FnCas12a; pFH16 and pFH46) and Lachnospiraceae bacterium (LbCas12a; pFH17 and pFH47) as well as with four related Cms1 nucleases (pFH18-21) (Additional file 2: Table S5). LbCas12a [22], FnCas12a [23] and Cms1 [24] have all been shown to work in plants.

Base editors are a rather recent addition to the range of available genome editing tools and allow targeted conversion of DNA base pairs as following: C-G to T-A [25] and A-T to G-C [26] without introducing a doublestrand break (DSB). The former base editor is based on the cytidine deaminase while the latter - on the adenosine deaminase. Both base editors have now been shown to be functional in various plants, including wheat, rice and tomato [27-31]. We have therefore generated level 0 modules encoding cytidine deaminase (pFH55 and pFH79) and adenosine deaminase (pFH45 and pFH92) based base editors (Additional file 2: Table S5).

EvolvR CRISPR-guided error-prone DNA polymerases have recently been shown to be able to introduce random point mutations at a targeted genomic locus [32]. Based on the Halperin et al. (2018) manuscript [32], we have generated a level 0 module with the wheat codon optimised version of enCas9-Poll3M-TBD (pFH77; Additional file 2: Table S5) that could prove to be a useful tool for reverse genetics in plants, particularly monocots.

We used the above mentioned CRISPR/Cas nuclease level 0 modules to assemble twenty-five nuclease expression units inserted into level $1 \mathrm{GG}$ vectors to be applied in monocot and dicot plant species (Additional file 2: Table S5).

\section{Guide RNA modules}

As CRISPR/Cas is an RNA-guided nuclease, guide RNA is its essential component that must be coexpressed with the nuclease in order to achieve ontarget DNA cutting. Guide RNAs are usually expressed under Pol III promoters, such as U3p or U6p, that have a defined transcription start nucleotide ('A' and 'G', respectively). A few genomic loci can be targeted simultaneously by CRISPR/Cas by coexpressing multiple guide RNAs and the modular cloning system is highly suitable for assembling constructs carrying multiple expression units, such as the CRISPR/Cas nuclease and guide RNAs.

As part of this study, we have generated a number of level 0 Pol III promoter modules (TaU3p, OsU3p, OsU6-2p and AtU6-26p; Additional file 2: Table S5). In addition, we have produced several guide RNA backbone level 0 constructs that can be used to assemble single or multiple guide RNA expression units without a PCR amplification step involved (Additional file 2: Table S5). The cloning system we present allows guide RNAs to be expressed either under individual Pol III promoters (Figs. 1, 2, 3; Additional file 1: section 2; Additional file 4: Figure S2) or from a polycistronic tRNA-sgRNA construct, which includes sgRNAs interspaced with tRNA scaffolds [6] (Figs. 4 and 5; Additional file 1: section 3; Additional file 4: Figure S3). The method with guide RNAs expressed under individual promoters enables expression of sgRNAs of the Cas9 family of CRISPR/ Cas nucleases, which carry the guide sequence at the $5^{\prime}$ end of sgRNA (Fig. 2), as well as crRNAs of Cas12a (Cpf1) nucleases and related Cms1 nucleases, which have the guide sequence at the $3^{\prime}$ end of crRNA (Fig. 3). Up to four guide RNAs under individual Pol III promoters can be assembled in using the former cloning procedure (Fig. 1b) and up to six sgRNAs per polycistronic construct - using the latter one (Fig. $4 \mathrm{~b}$ ). It should be noted that the number of guide RNAs under individual promoters could be increased to five, if no selectable marker is needed (Fig. $1 b)$, or many more if level $M /$ level $P$ vectors are used $[11,36]$. As to tRNA-sgRNA polycistronic constructs, the total number of sgRNAs assembled into a level 2 destination vector could be up to twenty-four (six per level 1), with a selectable marker, and up to thirty, if no selectable marker is used. Again, it is possible to add more than thirty sgRNAs by using level $\mathrm{M} /$ level $\mathrm{P}$ vectors $[11,36]$. Our GG toolkit enables the user to build such complex constructs within a week (Additional file 1: Figure S1). Flowchart diagrams illustrating pipelines of assembling level 2 constructs carrying gRNAs expressed under individual Pol III promoters, or as part of a polycistronic array, 
a

\section{Target specific annealed primers can be integrated into a level $\mathbf{0}$ acceptor vectors via Bpil}

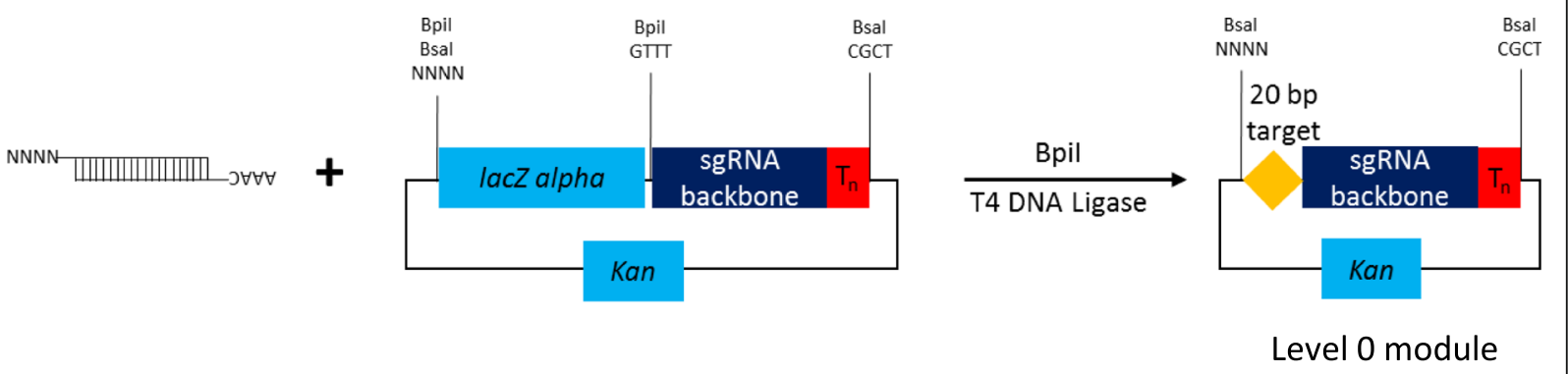

b

The gRNA module can be assembled with a Pol III promoter in a level 1 acceptor via Bsal

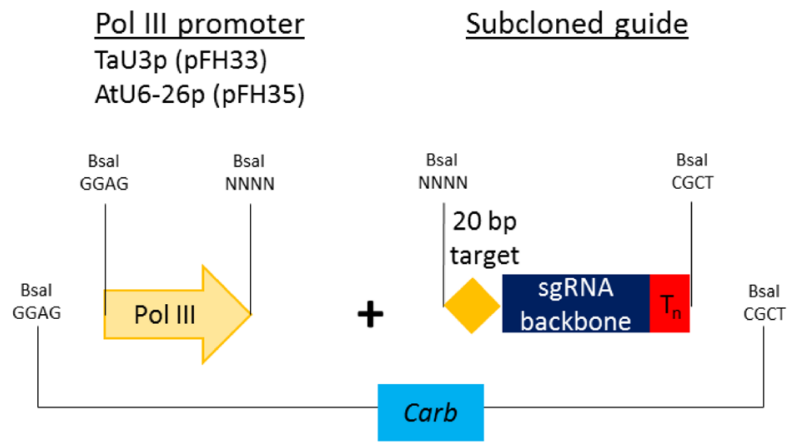

Level 1 backbone

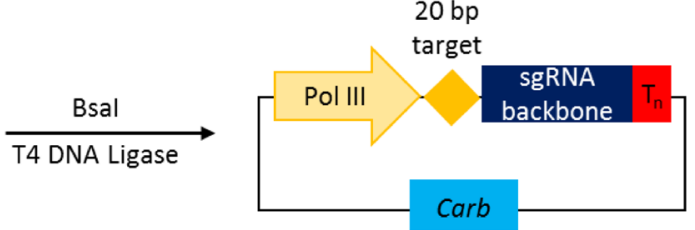

Level 1 module

Fig. 2 Assembly of level 1 sgRNA expression modules to be used with respective Cas 9 nucleases. During the first step (a) annealed complementary oligos encoding the guide are inserted into a level 0 acceptor with the sgRNA backbone using Bpil. During the second step (b), sgRNA is fused with the respective Pol III promoter using Bsal

can be found in Figs. S2 and S3 (Additional file 4), respectively.

\section{Testing of the tRNA-sgRNA CRISPR/Cas constructs in wheat protoplasts}

The GG toolkit therefore allows rapid parallel assembly of constructs by streamlining the cloning process. Since building multiple CRISPR constructs using GG is a straightforward procedure, it becomes reasonable to compare the activity of several experimental CRISPR setups in a transient expression system, such as protoplast, before proceeding with stable plant transformation, which could be a highly laborious and time-consuming process. Our CRISPR toolkit includes three wheat codon optimised SpCas9 versions (level 0 constructs pFH13, pFH24 and
pFH25; Additional file 2: Table S5) and their respective level 1 transcription units (pFH23, pFH66 and pFH67; Additional file 2: Table S5). These SpCas9 variants differ by e.g. nuclear localisation signal (NLS) versions or affinity tags. We have therefore compared the activity of the three Cas 9 variants in wheat protoplasts by cotransforming each of the level 1 constructs (pFH23, pFH66 and pFH67) with the level 1 plasmid containing the six sgRNAs (Fig. 6a) assembled into a tRNA-sgRNA array. This has allowed us to target three different wheat genes at once (Fig. 6a). We have targeted each gene by at least two sgRNAs with large deletions between Cas9 cut sites expected to be detectable by PCR due to DNA band shifts as previously described [4]. PCR amplification of the target genes has revealed clear 
a

Target specific annealed primers can be integrated into level 0 acceptor vectors via Bpil
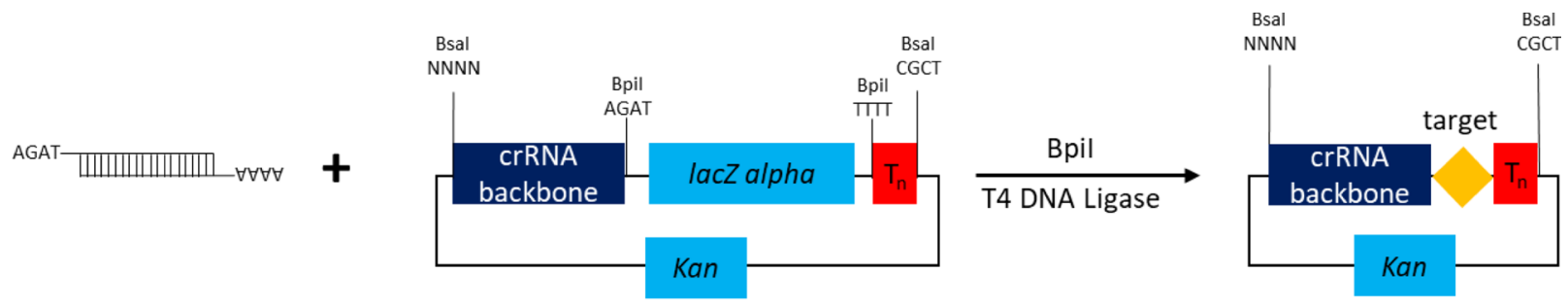

Level 0 module

b

The crRNA module can be assembled with a Pol III promoter in a level 1 vector via Bsal

Pol III promoter TaU3p (pFH33) AtU6-26p (pFH35) Subcloned guide

OsU6-2p (pFH37)

OsU3p (pFH39)

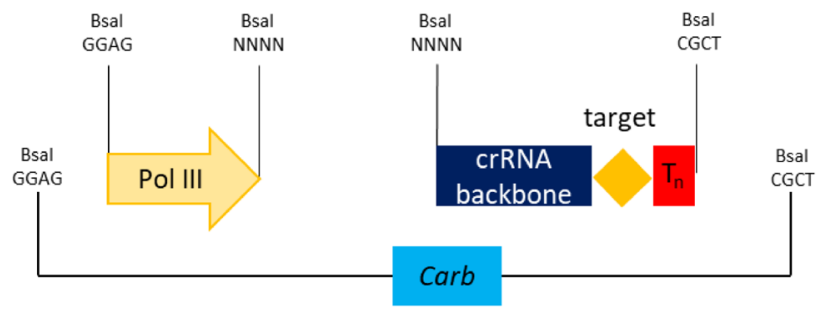

Level 1 backbone

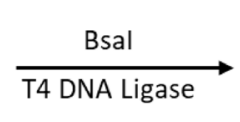

Level 1 module

Fig. 3 Assembly of level 1 crRNA expression modules to be used with respective Cas12a (Cpf1) and Cms1 nucleases. During the first step (a) annealed complementary oligos encoding the guide are inserted into a level 0 acceptor carrying the crRNA backbone using Bpil. During the second step (b), crRNA is fused with the respective Pol III promoter using Bsal

additional bands corresponding to alleles carrying large CRISPR/Cas-induced deletions in protoplasts transformed with pFH66. Results of Sanger sequencing of PCR amplicons from some of the shifted DNA bands can be found in Additional file 4: Figure S4 (.ab1 sequence trace files are available on Figshare, https://doi.org/10.6084/m9.figshare.11961975). In contrast, application of the other two Cas9 versions (pFH23 and $\mathrm{pFH} 67$ ) resulted in very faint bands of the size corresponding to amplicons carrying the deletions (Fig. 6b). Our results therefore suggest a significantly higher activity of the pFH66encoded SpCas9, as compared to the other two Cas9 variants, in wheat protoplasts.

\section{Discussion}

The modular cloning kit presented in the study enables quick and facile assembly of DNA constructs for genome editing applications in plants and is an addition to previously published collections of compatible GG modules [7,9-11]. The kit includes modules encoding a number of CRISPR/Cas nucleases (SaCas9, StCas9, LbCas12a etc.) that could be used as an alternative to the most commonly utilised SpCas9. SaCas9, for instance, has proven to be an efficient tool for generating gene knockouts in several plant species [15-18] and, in addition, has been shown to increase HDR efficiencies in plants [17]. Due to SaCas9 and StCas9 having longer PAM motifs than 


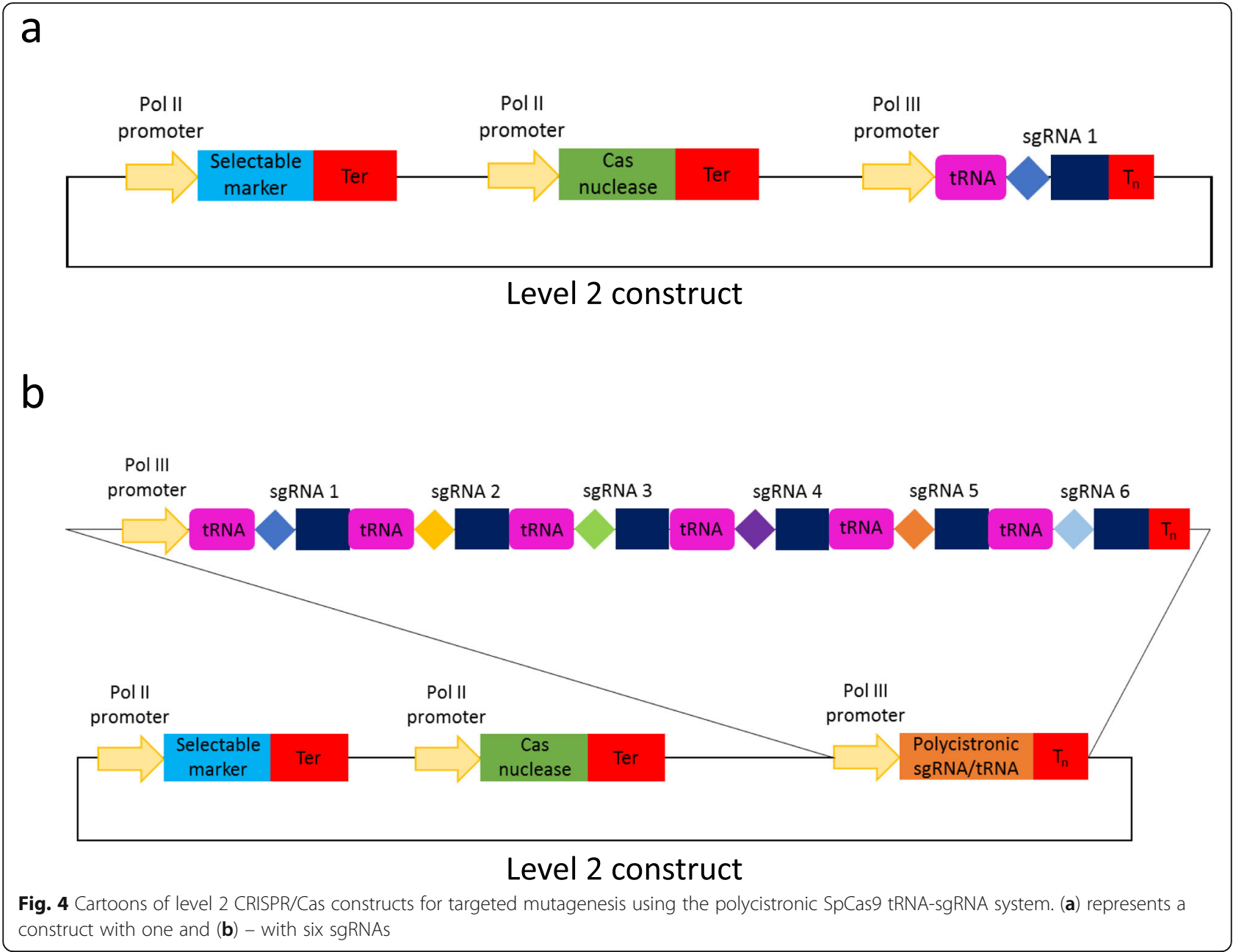

SpCas9 they are also likely to be more specific when it comes to DNA target recognition.

Cas12a (Cpf1) generates a staggered cut in DNA [37], while Cas9 - a blunt cut [14]. Due to this reason, Cas12a (Cpf1) could be a preferred choice of a CRISPR/Cas nuclease when it comes to HDR-based genome editing applications, such as targeted gene insertion [38, 39]. It is noteworthy that the modular cloning system is highly suitable for HDR-based applications as the DNA repair template could easily be cloned as a level 1 module into a level 2 destination vector. Also, since Cas12a (Cpf1) has a T-rich PAM motif ('TTTN' for LbCas12a (LbCpf1) and 'TTN' for FnCas12a (FnCpf1)), it could be a better choice for targeted mutagenesis in plant species with AT-rich genomes as compared to SpCas9 ('NGG' PAM).

As part of our study, we have generated a number of guide RNA backbone level 0 modules, which are compatible with respective Pol III promoters and CRISPR/ Cas nucleases (Additional file 1: Table S1). The guide RNA backbone modules can be used for PCR-free assembly of guide RNA expression units by cloning in an annealed pair of complimentary oligos encoding the guide sequence (Figs. 2 and 3). These guide RNA backbones are to be used when one wishes to express guide RNAs under individual promoters and up to four guide RNAs can be assembled into a level 2 vector together with level 1 modules encoding a CRISPR/Cas nuclease and a selectable marker (e.g. BAR, NPTII etc.; Fig. 1, Additional file 2: Table S5, Additional file 3: Table S6 and Additional file 4: Figure S2). We would also like to note that in addition to the PCR-free strategy for inserting the CRISPR/Cas9 guide sequence presented in this study (Fig. 2a), it is also possible to do it by PCRamplifying the sgRNA backbone (with the forward primer carrying the $20 \mathrm{bp}$ guide sequence at its $5^{\prime}$ end) and placing it under a Pol III promoter in a level 1 construct as previously described [8]. The latter strategy saves one cloning step but may not be suitable in the case of Cas12a whose crRNA backbone (around 40 nucleotides) could be too short for PCR amplification.

In addition to expressing each guide RNA under its own promoter, we have generated modules that allow assembly of polycistronic tRNA-sgRNA constructs with up to six sgRNAs expressed using a single Pol III 

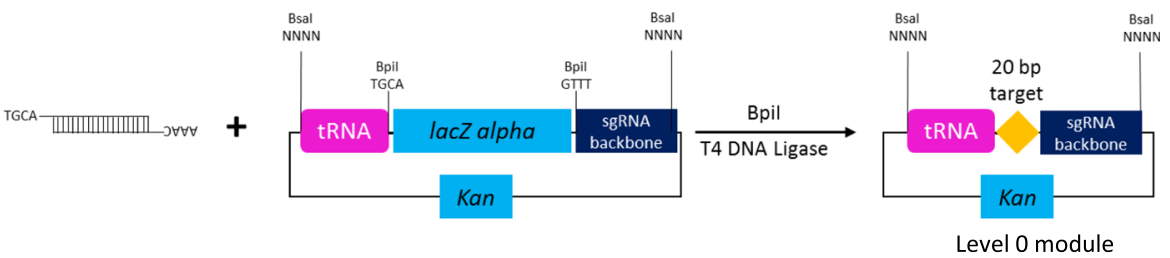

b Up to six sgRNA modules can be assembled into a level 1 acceptor vector via Bsal

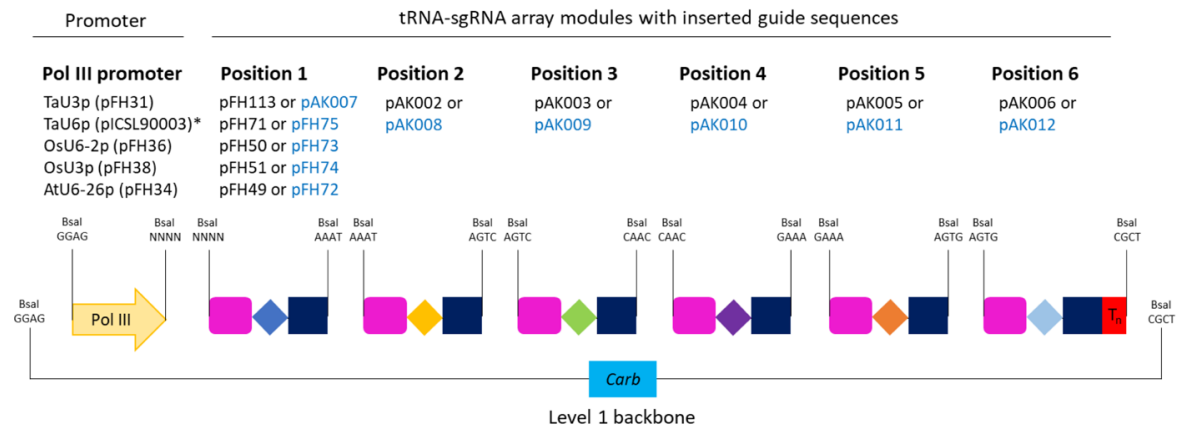

C Assembly of one to five tRNA-sgRNA modules into a level 1 vector requires endlinkers

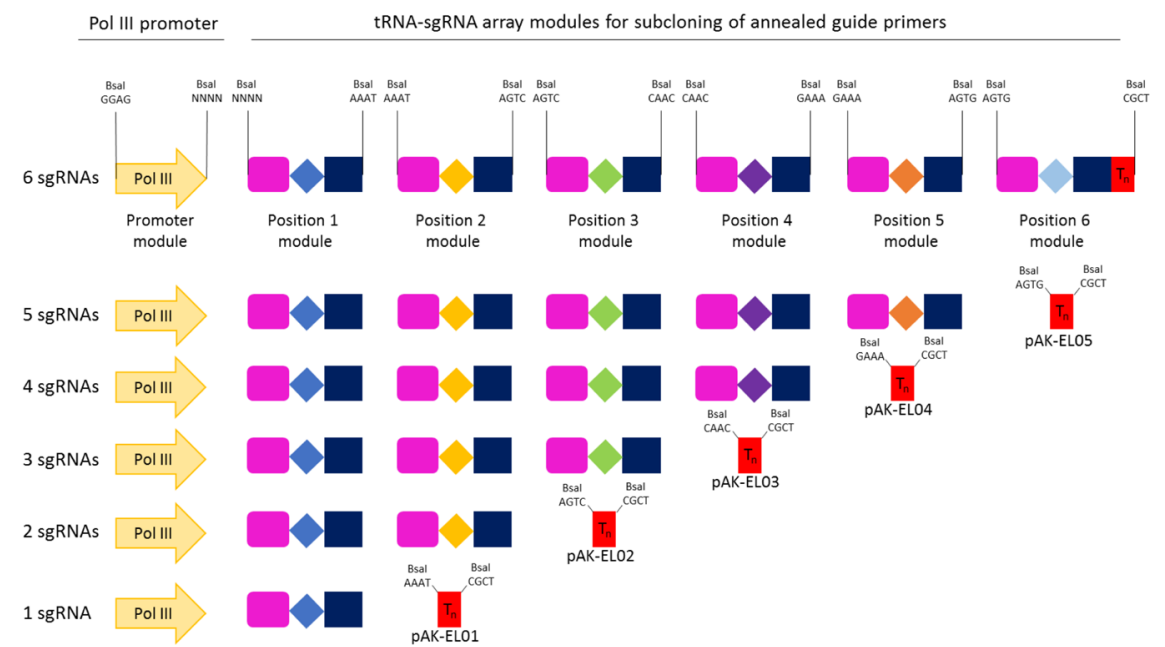

Fig. 5 Assembly of level 1 polycistronic tRNA-sgRNA expression modules to be used with SpCas9. During the first step (a) annealed complementary oligos encoding the guide are inserted into a level 0 acceptor with the tRNA-sgRNA backbone using Bpil. During the second step (b), tRNA-sgRNA modules are fused with the respective Pol III promoter using Bsal. Assembly of less than six tRNA-sgRNA modules into a level 1 vector requires respective endlinkers (c). pFH and pAK constructs shown in black font carry the improved sgRNA backbone [33], while the ones shown in blue font - the classic sgRNA backbone [34]. *This is a published module [35]

promoter (Fig. 4). The tRNA-sgRNA system, originally described by Xie et al. (2015) in rice, was later successfully applied in wheat [12] and Arabidopsis [13]. Nevertheless, the previously reported tRNA-sgRNA system relies on a rather cumbersome DNA construct assembly process as it involves PCR amplification of DNA fragments carrying repeats. The level 0 GG modules we have generated (Fig. 5 and Additional file 1: Table S3) enable straightforward and efficient assembly of tRNA-sgRNA arrays without a PCR step involved. The system offers a choice of five monocot and dicot Pol III promoters, with TaU6p being a published module [35], and two different SpCas9 sgRNA backbones (classic and improved; Additional file 1: Table S3).

As stable transformation continues to be a major bottleneck for genome editing applications in many 


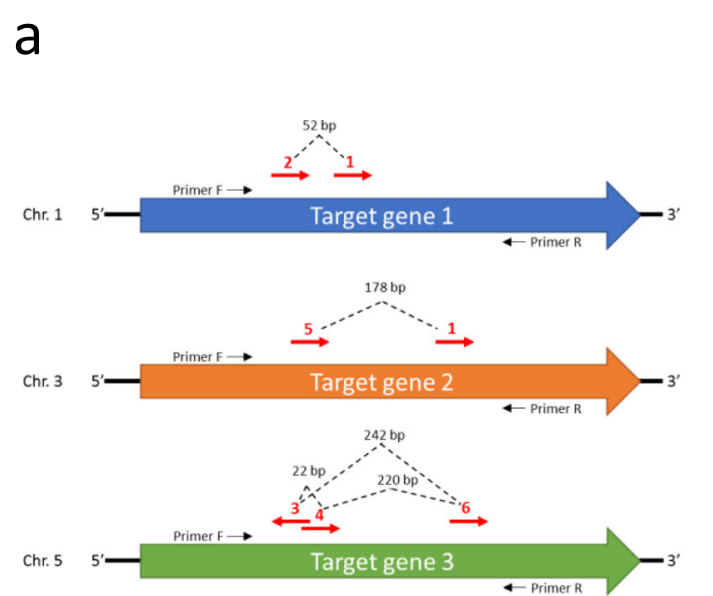

b

Fig. 6 Testing of tRNA-sgRNA CRISPR/Cas constructs in wheat protoplasts. Three wheat genes (a) were targeted: Target gene 1 (homoeologues
TraesCS1A02G338200, TraesCS1B02G350600 and TraesCS1D02G340400), Target gene 2 (homoeologues TraesCS3A02G289300, TraesCS3B02G323900
and TraesCS3D02G289100) and Target gene 3 (homoeologues TraesCS5A02G116500, TraesCS5B02G117800 and TraesCS5D02G129600). Red arrows
illustrate positions of the 20 bp sgRNA target sites. Dashed lines illustrate expected CRISPR/Cas-induced deletions. (b) Wheat protoplasts were Co-
transformed with a level 1 construct carrying one of the three SpCas9 variants (pFH23, pFH66 or pFH67) and the level 1 construct (pFH94)
carrying the six sgRNAs (a) in a tRNA-sgRNA array. Genotyping by PCR has revealed shifted DNA bands (marked by red asterisks) corresponding
to amplicons carrying CRISPR/Cas-induced deletions of expected sizes. The three panels shown come from different parts of the same DNA gel
(Additional file 4: Fig. S5). 'M' is the DNA marker; 'NTC' is the no template control

plants, including a major crop like wheat [40], it is advantageous to be able to test CRISPR/Cas constructs for activity in a transient expression system, such as protoplasts, before initiating an often lengthy and labour intensive stable transformation procedure. Using the wheat protoplast system, we have compared activity of three different wheat codon optimised SpCas9 variants (Fig. 6), which mostly differ in their C-terminal NLSs. The fact that one of the constructs (pFH66) performed better than the other two (Fig. 6b) could be due to the histone H2B NLS, located at the C-terminus of the pFH66 variant, being more efficient at importing Cas9 into the nucleus as compared to the SV40 or nucleoplasmin NLS present in the other two constructs. A possible link between different NLS versions and Cas9 activity was previously reported in Arabidopsis [9].

The tRNA-gRNA system for Cas9 multiplexing has proven to work in monocots $[6,12]$ as well as in dicots [13]. In dicots, it was shown that fusing tRNAs with the optimised sgRNA backbone [33] increased editing efficiencies [13]. In monocots however, only the classic sgRNA backbone [34] was so far used in tRNA-sgRNA arrays. The wheat protoplast assay has allowed us to verify the functionality of the tRNA-sgRNA array, carrying the optimised sgRNA backbone, in a monocot species. Our results suggest that the tRNA-sgRNA array, assembled using the optimised sgRNA backbone, could also result in higher CRISPR/Cas efficiencies in stably transformed monocot plants and, in particular, wheat. At the same time, we realise that the results obtained using protoplasts may not necessarily reflect the situation in stable transgenic lines due to differences in Cas9/sgRNA expression patterns as well as in ability to repair DNA.

\section{Conclusions}

We believe the presented modular cloning kit will become a valuable addition to the range of already available GG modules [7, 9, 10] and expect that plant researchers, working with both monocots and dicots, will find the presented molecular tools useful for various genome editing applications. We also believe our study will contribute towards wider adoption of the GG modular cloning system by plant researchers and consequently facilitate exchange of standardised molecular cloning parts across the research community.

\section{Methods}

\section{DNA construct assembly}

All PCR amplifications were performed using Q5 $5^{\circ}$ DNA Polymerase (New England Biolabs) according to the manufacturer's instruction. All GG cut-ligation reactions were performed according to the described protocol (Additional file 1: section 1).

All ligations were transformed into One Shot ${ }^{\mathrm{TM}}$ TOP10 chemically competent $E$. coli (Thermo Fisher Scientific) and constructs were verified by sequencing (Eurofins Genomics).

Specific details related to assembly of all GG modules reported in this study are provided (Additional file 1: section 4). Sequences of all PCR primers used in the 
study can be found in Table S4 (Additional file 1). All DNA constructs generated as part of this study were deposited with Addgene (www.addgene.org) with Addgene IDs indicated for each construct (Additional file 2: Table S5). The toolkit comprising 95 constructs will also be available from Addgene in a 96-well plate under the reference number 1000000159 . The rest of the materials (8 out of 103 constructs) can be requested by contacting the corresponding author. Sequence information of all constructs can be found in GenBank (.gb) files at www. addgene.org and on Figshare (https://doi.org/10.6084/ m9.figshare.11961975).

\section{Protoplast assay}

Protoplasts were isolated from etiolated 10 day old wheat seedlings (cv. Cadenza, sourced from CPB Twyford Ltd) as previously described [41] with some modifications. Cellulase R10 and Macerozyme R10 were obtained from Duchefa Biochemie (Haarlem, the Netherlands) and the enzymatic digestion was performed at $26^{\circ} \mathrm{C}$ for $4 \mathrm{~h}$. Subsequently, 50,000 protoplasts in a volume of $100 \mu \mathrm{L}$ were transformed with $20 \mu \mathrm{g}$ of each plasmid $(2 \mu \mathrm{g} / \mu \mathrm{L})$ purified using the Plasmid Maxi kit (Qiagen, Germany). One batch of protoplasts was treated with an equivalent amount of water and used as the negative (untransformed) control. Then, protoplasts were cultured in 6well plates for $60 \mathrm{~h}$. DNA was isolated and CRISPR/Casinduced mutations were detected by PCR amplification of the target genes using the DreamTaq DNA polymerase (ThermoFisher Scientific) and primer pairs FH432/ FH433 (Target gene 1), FH436/FH437 (Target gene 2) and FH440/FH441 (Target gene 3) (Additional file 1: Table S4). For sequencing, target genes were amplified using Q5 $5^{\circ}$ DNA Polymerase (New England Biolabs) according to the manufacturer's instruction with the same primer pairs as before. The PCR products were subcloned using the Zero Blunt ${ }^{\mathrm{Tm}} \mathrm{TOPO}^{\mathrm{m}}$ PCR Cloning Kit (Thermo Fisher Scientific). Single clones were Sangersequenced (Eurofins Genomics) using primer FH48 (Additional file 1: Table S4). Sequencing files were aligned and visualized using Geneious ${ }^{\ominus}$ version 10.2 (Biomatters).

\section{Supplementary information}

Supplementary information accompanies this paper at https://doi.org/10. 1186/s12870-020-02388-2

Additional file 1: Supplementary methods and protocols. Figure S1. A timeline for assembly of a level 2 CRISPR/Cas construct. Table S1. A list of modules encoding guide RNA backbones with matching CRISPR/Cas nucleases and Pol III promoters. Table S2. Primer design for inserting guide sequences into respective guide RNA backbone modules. Table S3. Pol III promoter modules and matching position 1 modules in the tRNA-sgRNA array. Table S4. Primers used in this study.
Additional file 2: Table S5. A list of GG constructs created during this study.

Additional file 3: Table S6. Additional GG constructs created by other groups.

Additional file 4: Figure S2. A flowchart diagram illustrating assembly of level 2 constructs with gRNAs expressed under individual Pol III promoters. Figure S3. A flowchart diagram illustrating assembly of level 2 constructs with gRNAs expressed as tRNA-sgRNA polycistronic units. Figure S4. Sanger sequencing reads illustrating gene deletions induced by CRISPR/Cas in wheat protoplasts. Figure S5. The unprocessed image of the DNA gel presented in Fig. 6b.

\section{Abbreviations}

CRISPR/Cas: Clustered regularly interspaced short palindromic repeats/ CRISPR-associated; crRNA: CRISPR RNA; DSB: Double-strand break; GG: Golden gate; HDR: Homology-directed repair; MoClo: Modular cloning;

PAM: Protospacer adjacent motif; sgRNA: Single guide RNA

\section{Acknowledgments}

We thank Lucy Hyde (University of Bristol, UK) for her contribution of managing the vector database and her help at establishing the protoplast protocol. We thank Mark Youles and Laurence Tomlinson (The Sainsbury Laboratory, Norwich, UK), and Sylvestre Marillonnet (Leibniz Institute of Plant Biochemistry, Halle, Germany) for the Golden Gate DNA constructs. We thank Robert Hoffie, Ingrid Otto and Nagaveni Budhagatapalli (IPK Gatersleben, Germany) for helpful advice on the protoplast assay.

\section{Authors' contributions}

VN conceived the idea of the manuscript; FH designed the pFH-constructs and performed the experiments; AK designed the PAK-constructs and assisted with cloning; LSL performed protoplast experiments together with $\mathrm{FH}$ and assisted with cloning; $\mathrm{VN}$ and FH wrote the manuscript. All authors read and approved the manuscript.

\section{Funding}

Vladimir Nekrasov (Rothamsted Research) receives grant-aided support from the Biotechnology and Biological Sciences Research Council (BBSRC) Designing Future Wheat (DFW) programme and Newton Fund. The authors declare that the funding bodies had no role in the design of the study and collection, analysis, and interpretation of data and in writing the manuscript.

\section{Availability of data and materials}

Most of the materials (DNA constructs) are available via Addgene (www. addgene.org) under individual reference numbers specified in Table S5 (Additional file 2). The toolkit comprising 95 constructs will also be available from Addgene in a 96-well plate under the reference number 1000000159. The rest of the materials ( 8 out of 103 constructs) can be requested by contacting the corresponding author. DNA construct sequence files are available in the GenBank format on www.addgene.org and Figshare (https://doi.org/ 10.6084/m9.figshare.11961975). Sanger sequence reads shown in Fig. S4 are available on Figshare (https://doi.org/10.6084/m9.figshare.11961975).

Ethics approval and consent to participate Not applicable.

Consent for publication

Not applicable.

\section{Competing interests}

The authors declare that they have no competing interests.

\section{Author details}

${ }^{1}$ Plant Sciences Department, Rothamsted Research, Harpenden AL5 2JQ, UK. ${ }^{2}$ Present address: John Innes Centre, Norwich Research Park, Norwich NR4 $7 \mathrm{UH}, \mathrm{UK}$. 
Received: 20 August 2019 Accepted: 6 April 2020

Published online: 23 April 2020

\section{References}

1. Belhaj K, Chaparro-Garcia A, Kamoun S, Patron NJ, Nekrasov V. Editing plant genomes with CRISPR/Cas9. Curr Opin Biotechnol. 2015;32:76-84.

2. Hahn F, Mantegazza O, Greiner A, Hegemann P, Eisenhut M, Weber APM. An efficient visual screen for CRISPR/Cas9 activity in Arabidopsis thaliana. Front Plant Sci. 2017;8. https://doi.org/10.3389/fpls.2017.00039.

3. Nekrasov V, Staskawicz B, Weigel D, Jones JD, Kamoun S. Targeted mutagenesis in the model plant Nicotiana benthamiana using Cas9 RNAguided endonuclease. Nat Biotechnol. 2013;31:691-3.

4. Nekrasov V, Wang C, Win J, Lanz C, Weigel D, Kamoun S. Rapid generation of a transgene-free powdery mildew resistant tomato by genome deletion. Sci Rep. 2017;7:482.

5. Hahn F, Eisenhut M, Mantegazza O, Weber APM. Homology-directed repair of a defective glabrous gene in Arabidopsis with Cas9-based gene targeting. Front Plant Sci. 2018;9. https://doi.org/10.3389/fpls.2018.00424.

6. Xie K, Minkenberg B, Yang Y. Boosting CRISPR/Cas9 multiplex editing capability with the endogenous tRNA-processing system. Proc Natl Acad Sci U S A. 2015;112:3570-5.

7. Weber E, Engler C, Gruetzner R, Werner S, Marillonnet S. A modular cloning system for standardized assembly of multigene constructs. PLoS One. 2011; 6:e16765.

8. Brooks C, Nekrasov V, Lippman ZB, Van Eck J. Efficient gene editing in tomato in the first generation using the clustered regularly interspaced short palindromic repeats/CRISPR-Associated9 system. Plant Physiol. 2014; 166:1292-7.

9. Castel B, Tomlinson L, Locci F, Yang Y, Jones JDG. Optimization of T-DNA architecture for Cas9-mediated mutagenesis in Arabidopsis. PLoS One. 2019; 14:e0204778.

10. Raitskin O, Schudoma C, West A, Patron NJ. Comparison of efficiency and specificity of CRISPR-associated (Cas) nucleases in plants: an expanded toolkit for precision genome engineering. PLoS One. 2019;14:e0211598.

11. Engler C, Youles M, Gruetzner R, Ehnert T-M, Werner S, Jones JDG, et al. A Golden Gate modular cloning toolbox for plants. ACS Synth Biol. 2014;3: 839-43.

12. Wang W, Pan Q, He F, Akhunova A, Chao S, Trick H, et al. Transgenerational CRISPR-Cas9 activity facilitates multiplex gene editing in allopolyploid wheat. The CRISPR Journal. 2018;1:65-74.

13. Zhang $\mathrm{Q}$, Xing $\mathrm{H}-\mathrm{L}$, Wang Z-P, Zhang $\mathrm{H}-\mathrm{Y}$, Yang $\mathrm{F}$, Wang $\mathrm{X}-\mathrm{C}$, et al. Potential high-frequency off-target mutagenesis induced by CRISPR/Cas9 in Arabidopsis and its prevention. Plant Mol Biol. 2018;96:445-56.

14. Jinek M, Chylinski K, Fonfara I, Hauer M, Doudna JA, Charpentier E. A programmable dual-RNA-guided DNA endonuclease in adaptive bacterial immunity. Science. 2012;337:816-21.

15. Kaya H, Mikami M, Endo A, Endo M, Toki S. Highly specific targeted mutagenesis in plants using Staphylococcus aureus Cas9. Sci Rep. 2016;6: 26871.

16. Steinert J, Schiml S, Fauser F, Puchta H. Highly efficient heritable plant genome engineering using Cas9 orthologues from Streptococcus thermophilus and Staphylococcus aureus. Plant J. 2015;84:1295-305.

17. Wolter F, Klemm J, Puchta H. Efficient in planta gene targeting in Arabidopsis using egg cell-specific expression of the Cas9 nuclease of Staphylococcus aureus. Plant J. 2018:94:735-46.

18. Schmidt C, Pacher M, Puchta H. Efficient induction of heritable inversions in plant genomes using the CRISPR/Cas system. Plant J. 2019;98:577-89.

19. Chatterjee P, Jakimo N, Jacobson JM. Minimal PAM specificity of a highly similar SpCas9 ortholog. Sci Adv. 2018;4:eaau0766.

20. Nishimasu H, Shi X, Ishiguro S, Gao L, Hirano S, Okazaki S, et al. Engineered CRISPR-Cas9 nuclease with expanded targeting space. Science. 2018;361: 1259-62.

21. Hu JH, Miller SM, Geurts MH, Tang W, Chen L, Sun N, et al. Evolved Cas9 variants with broad PAM compatibility and high DNA specificity. Nature. 2018;556:57-63.

22. Tang X, Lowder LG, Zhang T, Malzahn AA, Zheng X, Voytas DF, et al. A CRISPR-Cpf1 system for efficient genome editing and transcriptional repression in plants. Nature Plants. 2017;3:17018.

23. Endo A, Masafumi M, Kaya H, Toki S. Efficient targeted mutagenesis of rice and tobacco genomes using Cpf1 from Francisella novicida. Sci Rep. 2016;6: 38169.
24. Begemann MB, Gray BN, January E, Singer A, Kesler DC, He $Y$, et al. Characterization and validation of a novel group of type $v$, class 2 nucleases for in vivo genome editing. bioRxiv. 2017:192799. https://doi.org/10.1101/ 192799.

25. Komor AC, Kim YB, Packer MS, Zuris JA, Liu DR. Programmable editing of a target base in genomic DNA without double-stranded DNA cleavage. Nature. 2016;533:420-4.

26. Gaudelli NM, Komor AC, Rees HA, Packer MS, Badran AH, Bryson Dl, et al. Programmable base editing of $A \cdot T$ to $G \cdot C$ in genomic DNA without DNA cleavage. Nature. 2017;551:464-71.

27. Shimatani Z, Kashojiya S, Takayama M, Terada R, Arazoe T, Ishii H, et al. Targeted base editing in rice and tomato using a CRISPR-Cas9 cytidine deaminase fusion. Nat Biotech. 2017;35:441-3.

28. Zong Y, Wang Y, Li C, Zhang R, Chen K, Ran Y, et al. Precise base editing in rice, wheat and maize with a Cas9-cytidine deaminase fusion. Nat Biotech. 2017:35:438-40

29. Zong Y, Song Q, Li C, Jin S, Zhang D, Wang Y, et al. Efficient C-to-T base editing in plants using a fusion of nCas9 and human APOBEC3A. Nat Biotechnol. 2018;36:950-3.

30. Li C, Zong Y, Wang Y, Jin S, Zhang D, Song Q, et al. Expanded base editing in rice and wheat using a Cas9-adenosine deaminase fusion. Genome Biol. 2018;19:59.

31. Hua K, Tao X, Zhu J-K. Expanding the base editing scope in rice by using Cas9 variants. Plant Biotechnol J. 2019;17:499-504.

32. Halperin SO, Tou CJ, Wong EB, Modavi C, Schaffer DV, Dueber JE. CRISPRguided DNA polymerases enable diversification of all nucleotides in a tunable window. Nature. 2018;560:248-52.

33. Dang $Y$, Jia G, Choi J, Ma H, Anaya E, Ye C, et al. Optimizing sgRNA structure to improve CRISPR-Cas9 knockout efficiency. Genome Biol. 2015;16:280.

34. Mali P, Yang L, Esvelt KM, Aach J, Guell M, DiCarlo JE, et al. RNA-guided human genome engineering via Cas9. Science. 2013;339:823-6.

35. Lawrenson T, Shorinola O, Stacey N, Li C, Østergaard L, Patron N, et al. Induction of targeted, heritable mutations in barley and Brassica oleracea using RNA-guided Cas9 nuclease. Genome Biol. 2015;16:258.

36. Werner S, Engler C, Weber E, Gruetzner R, Marillonnet S. Fast track assembly of multigene constructs using Golden Gate cloning and the MoClo system. Bioengineered. 2012:3:38-43.

37. Zetsche B, Gootenberg JS, Abudayyeh OO, Slaymaker IM, Makarova KS, Essletzbichler $\mathrm{P}$, et al. Cpf1 is a single RNA-guided endonuclease of a class 2 CRISPR-Cas system. Cell. 2015;163:759-71.

38. Begemann MB, Gray BN, January E, Gordon GC, He Y, Liu H, et al. Precise insertion and guided editing of higher plant genomes using Cpf1 CRISPR nucleases. Sci Rep. 2017;7:11606.

39. Wolter $\mathrm{F}$, Puchta $\mathrm{H}$. In planta gene targeting can be enhanced by the use of CRISPR/Cas12a. Plant J. 2019;100:1083-94.

40. Sparks CA, Jones HD. Genetic transformation of wheat via particle bombardment. In: Henry RJ, Furtado A, editors. Cereal genomics: methods and protocols. Totowa, NJ: Humana Press; 2014. p. 201-18. https://doi.org/ 10.1007/978-1-62703-715-0_17.

41. Shan Q, Wang Y, Li J, Gao C. Genome editing in rice and wheat using the CRISPR/Cas system. Nat Protoc. 2014;9:2395-410.

\section{Publisher's Note}

Springer Nature remains neutral with regard to jurisdictional claims in published maps and institutional affiliations.

\section{Ready to submit your research? Choose BMC and benefit from:}

- fast, convenient online submission

- thorough peer review by experienced researchers in your field

- rapid publication on acceptance

- support for research data, including large and complex data types

- gold Open Access which fosters wider collaboration and increased citations

- maximum visibility for your research: over $100 \mathrm{M}$ website views per year

At $\mathrm{BMC}$, research is always in progress.

Learn more biomedcentral.com/submissions 